\title{
THE NETWORK ORGANIZATIONAL CHART AS A TOOL FOR MANAGING ORGANIZATIONAL COMPLEXITY
}

\author{
RAFAEL AITA \\ University of Lima, Peru.
}

\begin{abstract}
Companies in the twenty first century are required to continually adapt to an uncertain and ever-changing environment, which requires a more organic and flexible organization, without losing the benefits of volume production and economies of scale. The Organizational Network is a tool that allows the company to study the channels through which materials, money, and information resources flow between jobs, unlike the traditional Organizational Chart that only shows the hierarchy of power. Analyzing these links like a complex network will allow the manager to identify bottlenecks and agglomerations of power and measure the entropy generated in the organization. This gives a quantitative measure of the complexity of the business and, therefore, both its organic growth and efficient growth can be managed.

Keywords: chaos theory, complexity, entropy organization, information, strategical planning, uncertainty.
\end{abstract}

\section{THE LINEAR MODEL}

The Industrial Revolution introduced a series of social and economic changes that shaped the world of the nineteenth and twentieth centuries, beginning the 'industrial society'. The structure of industries in this society was consolidated thanks to the theories of Henri Fayol, Max Weber, and Frederick Taylor who laid the foundations to abandon handcrafted and empirical work.

A century later, we find ourselves again facing a new conjuncture of change due to the emergence of the new Information Technologies that has brought a significant increase in the volatility of the market and in acceleration of change. Faced with this new situation, the question arises whether we should continue to see companies in the traditional way or should we consider it as a complex system that requires non-linear tools to be managed. Management books provide a response by predicting the birth of a new management theory while they see the need for it. So says Gary Hamel [1]: 'Could the exercise of the management change in a manner just as radical during the first two or three decades of this century as it did during the early years of the twentieth century? I believe so. More than that, I think we should make it so'. Faced with this new reality, the principles of the classical and neoclassical administrative theories become obsolete, leaving the executives with poor management tools for business that need to adjust to the characteristics of a volatile market.

\section{THE COMPLEXITY IN THE COMPANY}

However, the question how to model the complexity in the company still remains. Although the traditional scheme that synthesizes relations in a company hierarchy is the organizational chart, this tool fails to collect the interrelationships of exchange of information, materials, and money between the different job positions. It is these relationships that are precisely the source of greater complexity. To model this emerging complexity in the company, it is intended to take the organizational chart as

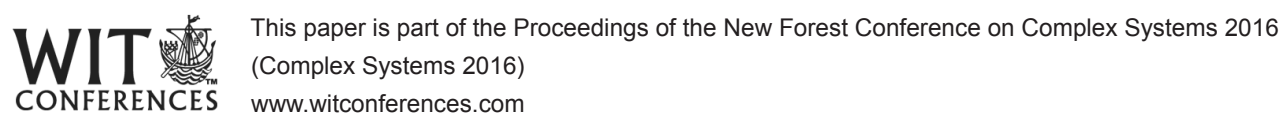

(C) 2016 WIT Press, www.witpress.com

ISSN: 1755-7437 (paper format), ISSN: 1755-7445 (online), http://www.witpress.com/journals DOI: 10.2495/DNE-V11-N4-593-599 
a three-dimensional object which needs to be observed from a top view rather than from a front view. This new perspective allows to show the relationships of communication and interaction between each position forming a complex network instead of a hierarchical structure. According to Aldana [2], complex networks are sets of many connected nodes that interact in some way. The nodes of a network, also called vertices or elements, are represented by the symbols $v_{1}, v_{2}, \ldots v_{n}$, where $n$ is the total number of nodes in the network. If $a v_{i}$ node is connected to $a v_{j}$ node, this connection is represented by the ordered pair $\left(\mathrm{v}_{\mathrm{i}}, \mathrm{v}_{\mathrm{j}}\right)$. This connection is known as an 'edge' within the network. In the case of the organization, nodes are given by the job positions and the edges by the relations between these positions. The relationship between positions should not be linked arbitrarily, but among those positions where there is a real exchange of material and financial resources, or the most important resource of this era: information. Therefore, the connections must be drawn based on the connections that exist in the Process Diagrams of the Company. Process diagrams show the relationship between of exchange of information corresponding to each function of the company. In summary, the Network Diagram of the Organization is an $\mathrm{R}$ complex network consisting of a set of nodes $\mathrm{V}=\left\{\mathrm{v}_{1}, \mathrm{v}_{2}, \ldots \mathrm{v}_{\mathrm{n}}\right\}$ and a set of ordered pairs $\mathrm{E}=\left\{\left(\mathrm{v}_{\mathrm{i}}, \mathrm{v}_{\mathrm{j}}\right)\right\}$, which belongs to $\mathrm{V} \times \mathrm{V}$ and for its construction, it is powered by two diagrams: the organizational chart to draw the network nodes (positions of the Company) and the Process Diagrams to draw the edges that connect the nodes according to each link that exists in the process.

Finally, it is necessary to emphasize that the links between nodes are no communication channels. If so, all positions of the company would be connected to each other, as any person can communicate verbally or through e-mail with any other employee of the company. The links between exchange of information should only display those information flows that occur continuously as part of the production process of goods or services of the company and not from a casual or sporadic interaction. For this reason the links of interaction should be taken from the Process Diagrams of the Company and must explicitly appear in it. The complex network of the organization is a non-directed network, since for each pair $\left(\mathrm{v}_{\mathrm{i}}, \mathrm{v}_{\mathrm{j}}\right)$ belonging to $\mathrm{E}$ there is also the pair $\left(\mathrm{v}_{\mathrm{j}}, \mathrm{v}_{\mathrm{i}}\right)$ accordingly.

The advantage of focusing the organization from a Network Diagram is that it allows analyzing the company as a complex network and, therefore, helps in viewing the degree of connectivity between its elements, the level of integration between areas, and the formation of islands or people who centralize information. Figure 1 shows an example of the Organizational Network of a company of Information Technology based on the chart in Fig. 2.

\section{THE CONNECTED COMPANY}

To outline the organization as a network, the first thing you may notice is that not all nodes are necessarily connected, so that not all nodes have the same number of links within the network. The number $\mathrm{k}_{\mathrm{i}}$ of neighbors of the node $\mathrm{v}_{\mathrm{i}}$ (the number of connections of $\mathrm{v}_{\mathrm{i}}$ ) is called the $\mathrm{v}_{\mathrm{i}}$ connectivity and the average of these connectivities in all the nodes is called the network connectivity. The connectivity index defines the degree to which the network is connected and is calculated according to the formula:

$$
\text { Index of network connectivity }=\frac{\text { sum of links }}{\text { sum of nodes }}
$$

The advantages of a network with a greater degree of connectivity are as follows:

- Increased robustness of the network: The structural strength of the network is defined by Aldana [2] as the invariance of phenotypes in the presence of internal or external disturbances to the 


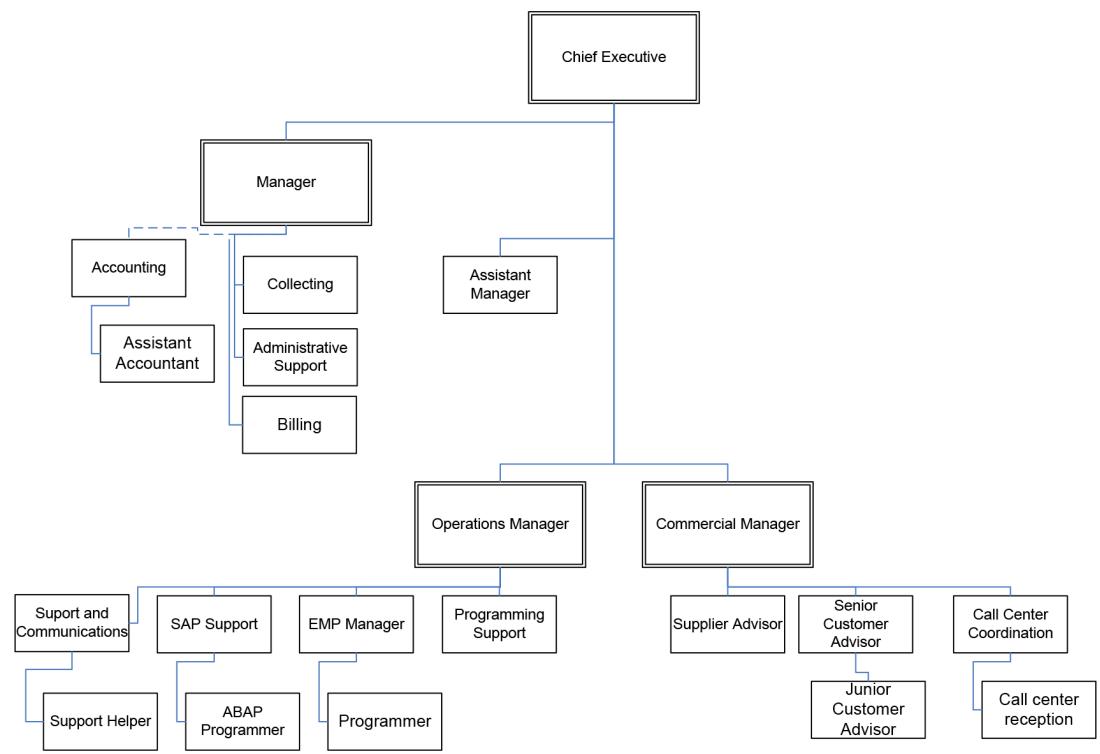

Figure 1: Organizational chart or network diagram of the organization.

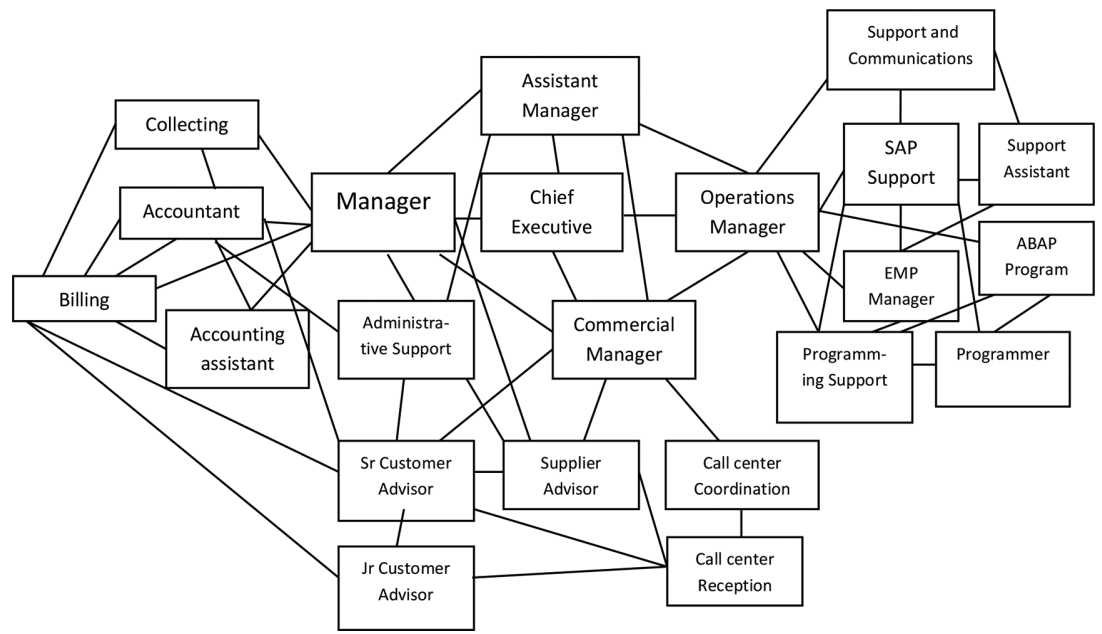

Figure 2: Organizational Chart of an IT Company.

organism. In this case it would measure the capacity of the enterprise network to re-adapt after one of the nodes (workstation) is either temporarily or indefinitely disabled. A useful analysis for the Human Resources department to anticipate the consequences of sudden resignation, absenteeism, or job redesign.

- Enhanced communicability of the network: The minimum length between two nodes is defined as the minimum number of edges that must be passed to get from one node to another node of 
the network. The average length of the network is the average of minimum lengths between all possible pairs of nodes of the network. A higher network connectivity offers a greater number of minimum lengths within the network and thus reduces the average length of the network. This translates as a greater horizontality in the organization, less bureaucracy in the communication, and greater closeness between the strategic apex with the rest of the company.

- Spread of information on the network: Rosvall, Trusina, Minhagen, and Sneppen et al. [3] proposed that a complex network structure is linked to its reliability and the speed of propagation of information, defining the entropy of the network as the degree of difficulty in seeking information on the network.

- Highest degree of network evolution: The size of the network does not end with the organization's boundary vertices, since these are connected with customers who are at the same time connected with other's customers or non-customers. This means that the network is expanding, not only internally through the incorporation of new job positions, but also externally. Therefore, the growth of the network will be studied as part of the market growth.

\section{NETWORK ROBUSTNESS}

Rodrigues, da F. Costa et al. [4] propose a method to determine the vulnerability of a network looking for its most vulnerable vertices. The vulnerability of a vertex can be defined according to the drop in network performance when the vertex, with all its edges, is removed. The vulnerability of the network would be equivalent to its most vulnerable vertex. The analysis of disturbances consists in measuring changes in the network to the non-use of their different vertices and helps to determine the robustness of the network against external shocks.

In the case of the Network flow chart in Fig. 1, the index of network connectivity will be measured before and after removing a job. The position that, at the time of being withdrawn, registers a further decline in the index of network connectivity will be the one that determines the degree of vulnerability of the network. To calculate this, Table 1 shows the indexes of the network connectivity of Fig. 1 before and after removing the designated positions.

The main conclusion that is drawn from the results of Table 1 is that there is little dispersion between the degree of variation when removing each of the nodes. This means that there is no centrality in a specific position and the network is homogenously interconnected. It also highlights that the absence of the Chief Executive position represents a low variation in network connectivity. This

Table 1: Robustness of the network.

\begin{tabular}{lccc}
\hline Position to be withdrawn & $\begin{array}{c}\text { Original connectivity } \\
\text { index }\end{array}$ & $\begin{array}{c}\text { Connectivity index } \\
\text { without the position }\end{array}$ & \% Variation \\
\hline Manager & 2.41 & 2.09 & 13.27 \\
Chief executive & 2.41 & 2.33 & 3.31 \\
Commercial manager & 2.41 & 2.19 & 9.12 \\
Operations manager & 2.41 & 2.14 & 11.2 \\
Adminstrative support & 2.41 & 2.28 & 5.39 \\
SAP support & 2.41 & 2.23 & 7.46 \\
ERP manager & 2.41 & 2.23 & 7.46 \\
\hline
\end{tabular}


means that the senior management policy is based on delegating tasks to subordinates. An eventual absence of the Chief Executive may be supplied between the functions of the other managers without compromising the structure of the network. Finally, the vulnerability of the network lies in the position of the manager, which is the most connected in the company, lies in the position of Manager. This result shows that it is not necessary that a position belongs to the senior management to have a significance in network connectivity, which until now has been listed as an informal organization within the company, but which, with this tool, can be identified and quantified.

The index of network connectivity can be misleading, since not all generated links have the same quality. In 1976, Price proposed the model of preferential attachment. Based on the number of quotations from scientific literature, he deduced that the probability that an article is cited is directly proportional to the number of quotations it already has. The preferential attachment indicates that nodes with lots of connections will prefer to connect with other nodes that have large numbers of connections. Thus, higher quality links are generated in the network.

\section{SPREAD OF INFORMATION IN THE NETWORK}

The measure of information is the entropy, which led Claude Shannon and Weaver [5] to design a Mathematical Information Theory based on the measurement of entropy. In a complex network, entropy measures the difficulty of spreading information in the network and, therefore, it is an index to measure the level of bureaucracy in the organization. Rosvall, Trusina, Minhagen, and Sneppen et al. [3] proposed to quantify the information associated to locate a specific target in the network by measuring the probability of taking the shortest path from vertex $i$ to vertex $b$ in a random choice through the formula:

$$
\mathrm{P}[\mathrm{p}(\mathrm{i}, \mathrm{b})]=\frac{1}{k_{i}} \prod_{\mathrm{j} \in \mathrm{p}(\mathrm{i}, \mathrm{b})} \frac{1}{k_{j}-1}
$$

where $\mathrm{P}$ is the probability to take the shortest path $\mathrm{p}(\mathrm{i}, \mathrm{b})$ from vertex $\mathrm{i}$ to vertex $\mathrm{b}$ and $\mathrm{k}_{\mathrm{j}}$ is the degree of vertex $j$, and the product includes all vertices $j$ on the path $p(i, b)$ excluding $i$ and $b$ themselves. The amount of information used in the search to find the shortest path between $\mathrm{i}$ and $\mathrm{b}$ is given by the entropy $S(i, b)$ :

$$
\mathrm{S}(\mathrm{i}, \mathrm{b})=-\log _{2} \sum_{\{\mathrm{p}(\mathrm{i}, \mathrm{b})\}} \mathrm{P}[\mathrm{p}(\mathrm{i}, \mathrm{b})]
$$

Where the sum is taken from all shortest paths $\mathrm{p}(\mathrm{i}, \mathrm{b})$ from $\mathrm{i}$ to $\mathrm{b}$.

In the case of the network shown in Fig. 1, we can calculate the entropy from an order that comes from the Chief Executive and goes up to the programmer searching for the probability of finding the shortest path in a random choice between these two vertices in the network.

There are three shorter paths between the Chief Executive and the programmer and they are:

1. Chief Executive - Operations manager - Programming support - Programmer

2. Chief Executive - Operations manager - SAP Support - Programmer

3. Chief Executive - Operations manager - ABAP Programming - Programmer

The probability of finding the shortest path following route 1 is $=0.892 \%$

The probability of finding the shortest path following route 2 is $=0.714 \%$

The probability of finding the shortest path following route 3 is $=1.785 \%$

The entropy of the three paths equals to: $-\log _{2}(0.00892+0.00714+0.01785)=4.8818$ bits. 
That is the amount of information lost in delivering a message from the Chief Executive to the Programmer.

The total entropy of the organization would be equivalent to the sum of the entropies of all pairs of nodes. This result depends on the index of connectivity of the company, but paradoxically, in this case, a greater connectivity of the company generates a greater entropy. The more the paths between nodes, the lower the probability of finding the shortest path in a random choice and, therefore, a greater entropy and loss of information travels over the network. That is why the nodes with greater connectivity, in this case the SAP Support, are the ones that add more entropy. This is in contradiction to the conclusion of the preceding paragraph, where a greater connectivity granted greater robustness to the network and resistance to the disqualification of a node. Is there a possibility of increasing connectivity and, thereby, the network robustness, without significantly increasing the entropy?

\section{CLUSTERING}

Clustering is the intermediate response between the increase of the connectivity through the number of links and the location of the optimal way to distribute information. Clustering consists in increasing the connectivity in concentrated clusters of nodes in order to strengthen their robustness, but not across the network to avoid significantly increasing the entropy thus, optimizing the likelihood of increasing the shortest path between two points practically until the end of the line where the cluster is located. It is important to highlight that, historically, the grouping of functions in departments, areas, and business units have followed this principle of clustering, demonstrating a greater organizational efficiency even beyond the company as evidenced by the industrial clusters proposed by Michael Porter. However, this principle of clustering should not necessarily be limited to business areas, but it can also be applied to functions that are close in Process Diagrams rather than the organizational chart. This is an example of the multidisciplinary work teams that so successfully brought to organizations that specialize in the development of projects.

The level of clustering in a complex network is measured through the coefficient of aggregation, which is defined as the probability that two nodes connected to a third node, are, in their turn, connected among themselves. This probability is measured through the transitivity of the network, which is defined by Newman [6] as the presence of a larger number of triangles in the network, being the triangle, the set of three vertices where each is connected to each of the others. Likewise, da F. Costa et al. [4] presented the formula to calculate the level of clustering C:

$$
\mathrm{C}=\frac{3 \mathrm{~N}_{\Delta}}{\mathrm{N}_{3}}
$$

Where $\mathrm{N}_{\Delta}$ is the number of triangles in the network and $\mathrm{N}_{3}$ is the number of triples connected. A triangle is a set of three vertices with edges between every pair of vertices.

If $\mathrm{k}_{\mathrm{i}}$ is the number of neighbors of the vertex $\mathrm{i}$, then $\mathrm{N}_{3}(\mathrm{i})=\mathrm{k}_{\mathrm{i}}\left(\mathrm{k}_{\mathrm{i}}-1\right) / 2$. In addition, if $\mathrm{N}_{\Delta}(\mathrm{i})$ counts the number of edges between neighbors of $i$ and the number of edges are represented between neighbors of $\mathrm{i}$ as $\mathrm{L}_{\mathrm{i}}$, then the equation to calculate the level of clustering $\mathrm{C}$ can be rewritten as:

$$
\mathrm{C}=\frac{2 \mathrm{~L}_{\mathrm{i}}}{\mathrm{k}_{\mathrm{i}}\left(\mathrm{k}_{\mathrm{i}}-1\right)}
$$

\section{CONCLUSIONS}

The Network Organizational Chart is a tool that uses complex networks to study the exchange of materials, money, and information between the different positions of the company. The network 
nodes represent the job positions and are taken from the organizational chart of the company, while the edges represent the relationships between those positions according to the flows that appear in the Process Diagrams of the company. Analyzing the organization as a complex network allows to study, among other things, the centrality of power, the robustness of the organization, and the spread of information through the company.

The companies show an informal organization that emerges from the relationship among the job positions that do not appear on the organizational chart. The Network Organizational Chart may determine the centrality of the job positions according to the network and detect the focus of power.

The robustness of the organization is determined by the invariability that the network shows after any of its nodes is withdrawn. This robustness can be increased by increasing the number of links within the network and is measured through an indicator called the index of connectivity.

The efficiency of the transmission of the information by the network is calculated based on the probability of finding at random the shortest path between two nodes and is quantified through the entropy of the network. With increased connectivity of the network, the lower the probability of finding the shortest path and, therefore, a greater entropy.

To increase the robustness of the network without significantly increasing the entropy, the connectivity in targeted clusters can be increased, which is known as clustering and it is this principle that governs the division of the organization into departments and areas. In the complex network, clustering is measured through the index of clustering, which, together with the entropy and the rate of connectivity, provides the guidelines for an optimal organizational design of the company.

\section{REFERENCES}

[1] Hamel, G. \& Breen, B., The Future of Management, Harvard Business Press, 2013.

[2] Aldana, M., Complex networks. Taken from de available at http://www. fis. unam. $\mathrm{mx} / \sim \max /$ English/notasredes.pdf, 2006

[3] Sneppen, K.. Trusina, A. \& Rosvall, M., Measuring information networks. Pramana, 64(6), pp. 1121-1125, 2005. http://dx.doi.org/10.1007/BF02704173

[4] Costa, L.D.F., Rodrigues, F.A., Travieso, G. \& Villas B.P.R., Characterization of complex networks: a survey of measurements. Advances in Physics, 56(1), pp. 167-242, 2007. http://dx.doi.org/10.1080/00018730601170527

[5] Shannon, C.E. \& Weaver, W., The mathematical theory of information, 1949.

[6] Newman, M.E., The structure and function of complex networks. SIAM Review, 45, pp. 167256, 2003.

http://dx.doi.org/10.1137/S003614450342480 